\title{
Repercussões do abuso e exploração sexual na criança e adolescente e a importância da qualificação da enfermagem frente aos casos: revisão narrativa
}

\author{
Repercussions of sexual abuse and exploitation in children and adolescents and the \\ importance of nursing qualification and notification in cases: narrative review
}

Repercusiones de abuso y explotación sexual en niños y adolescentes y la importancia de la calificación y notificación de enfermería en los casos: revisión narrativa

Emilie dos Santos Aleluia1 , Maria Ledijane Reis de Carvalho', Vitoria Vilas Boas da Silva Bomfim", Mariza Araujo dos Santos Dias ${ }^{1}$, Victor de Matos Leal ${ }^{1}$, Aglaya Oliveira Lima Cordeiro de Almeida ${ }^{1}$, Ana Flora Sena Santa Fé2, Rebeca Pereira da Rocha².

\section{RESUMO}

Objetivo: analisar de acordo com a literatura as repercussões do abuso e exploração sexual na criança e adolescente bem como destacar a importância da qualificação profissional da enfermagem e das notificações frente aos casos. Revisão bibliográfica: $O$ abuso e exploração sexual infantil consistem na violação dos direitos sexuais de crianças e adolescentes de forma não consensual e impositiva, envolvendo essa população em atividades sexuais inapropriadas para sua maturidade física e psicológica trazendo diversas repercussões na vida do indivíduo, sendo apontada como uma das principais causas de morbimortalidade no Brasil tornando-se grande problema de saúde pública. Diante disso constatou-se a importância da enfermagem na abordagem do abuso sexual, visto que é a maior equipe de uma unidade de saúde, assumindo o papel de protagonismo na abordagem do tema, requerendo assim conhecimento científico e domínio para notificar os casos de forma precisa. Considerações Finais: $O$ abuso e a exploração sexual infanto-juvenil causam severos distúrbios comportamentais e emocionais na vítima que vão desde abuso de substâncias psicoativas ao pensamento autodestrutivo. É essencial e urgente a qualificação do enfermeiro, visto que há um descompasso entre a atuação de enfermagem para identificar e notificar os abusos com as reais necessidades do menor.

Palavras-chave: Enfermagem, Abuso sexual, Infância, Adolescência.

\section{ABSTRACT}

Objective: To analyze, according to the literature, the repercussions of sexual abuse and exploitation on children and adolescents, as well as to highlight the importance of professional nursing qualification and notifications regarding cases. Bibliographic review: Child sexual abuse and exploitation consists of violating the sexual rights of children and adolescents in a non-consensual and imposing manner, involving this population in inappropriate sexual activities for their physical and psychological maturity, bringing several repercussions on the individual's life, being pointed out as one of the main causes of morbidity and mortality in Brazil, becoming a major public health problem. Therefore, the importance of nursing in addressing sexual abuse was observed, since it is the largest team in a health unit, assuming the leading role in addressing the theme, thus requiring scientific knowledge and domain to accurately notify cases. Final considerations: Child abuse and sexual exploitation of children cause severe behavioral and emotional disorders in the victim, ranging from substance abuse to self-destructive thinking. It is essential and urgent to qualify the nurse, as there is a mismatch between the nursing work to identify and notify abuses with the real needs of the child.

Key words: Nursing, Sexual abuse, Childhood, Adolescence.

${ }^{1}$ Centro Universitário Jorge Amado (UNIJORGE), Salvador - BA. *E-mail: emilie.aleluia@gmail.com ${ }^{2}$ Hospital Português, Salvador - BA. 


\section{RESUMEN}

Objetivo: Analizar, de acuerdo con la literatura, las repercusiones del abuso y la explotación sexual en niños y adolescentes, así como resaltar la importancia de la calificación y las notificaciones de enfermería profesional en relación con los casos. Revisión bibliográfica: El abuso y explotación sexual infantil consisten en violar los derechos sexuales de niños y adolescentes de manera no consensuada e imponente, involucrando a esta población en actividades sexuales inapropiadas para su madurez física y psicológica, lo que trae varias repercusiones en la vida del individuo, señalando como Una de las principales causas de morbilidad y mortalidad en Brasil, convirtiéndose en un importante problema de salud pública. Por lo tanto, se observó la importancia de la enfermería para abordar el abuso sexual, ya que es el equipo más grande en una unidad de salud, asumiendo el papel principal en el tratamiento del tema, lo que requiere conocimiento científico y dominio para notificar con precisión los casos. Consideraciones finales: El abuso y la explotación sexual de niños y adolescentes causan graves trastornos conductuales y emocionales en la víctima, que van desde el abuso de sustancias hasta el pensamiento autodestructivo. Es esencial y urgente calificar a la enfermera, ya que existe un desajuste entre el trabajo de enfermería para identificar y reportar abusos con las necesidades reales del niño.

Palabras clave: Enfermería, Abuso sexual, Infancia, Adolescencia.

\section{INTRODUÇÃO}

O abuso sexual do menor acontece quando seu corpo é usado para satisfação sexual de alguém que se utiliza da relação de autoridade, incluindo desde a prática de carícias, manipulação de genitálias, mamas ou ânus, pornografia, até o ato sexual com ou sem penetração, com ou sem o uso de violência física. Tratado pela Organização mundial de Saúde como um grande problema de saúde pública (WHO, 2003). Já a exploração sexual significa usar este menor para atividade sexual remunerada, representa uma das piores formas de violação humana, promovendo uma relação desigual de poder que retrata o controle e a violência que se instalam na vida das vítimas (LIBÓRIO R, 2005; SERPA MG, 2010).

As pesquisas brasileiras sobre violência sexual contra crianças e adolescentes revelam que todas as faixas etárias são vítimas, em sua maioria entre nove e doze anos de idade, com predominância do sexo feminino. Sendo os agressores pessoas conhecidas, do convívio intrafamiliar e que a casa da vítima ou do autor é o local predominante dos abusos. Configurando uma barreira privada que dificulta as intervenções de medidas de prevenção contra o abuso e até mesmo medidas de punições aos abusadores (SILVA MAI e FERRIANI MGC, 2007).

As repercussões desses abusos sexuais têm se tornado um problema de saúde pública, uma vez que atinge diversas famílias brasileiras (GUIMARÃES JATL e VILLELA WV, 2011). Tendo em vista que a violência contra crianças e adolescentes é considerada problema de saúde pública, o Ministério da Saúde (MS) criou estratégias com o intuito de fortalecer a atuação dos profissionais de saúde para a importância da integralidade do cuidado em todas as suas dimensões: acolhimento, atendimento, notificação e seguimento na rede de cuidados e de proteção social (BRASIL, 2018).

Os dados epidemiológicos comprovam o aparecimento de inúmeros casos a cada ano. Adepto ao panorama de subnotificação desses casos, a falta de sistematização das informações, o despreparo dos profissionais e de padronização das ferramentas para investigação/abordagem contribuem para a dificuldade em se obter dados válidos (LEITE JT, et al., 2016).

O boletim epidemiológico número 49 de junho de 2018, retratou sobre o perfil epidemiológico das violências sexuais notificadas contra crianças e adolescentes no período de 2011 a 2017 . Totalizaram neste período 1.460 .326 crianças e adolescentes que sofreram violência interpessoal, coletiva ou autoprovocada, destes 184.524 casos de violência sexual. Observa-se neste período um grande aumento geral de casos notificados de violências sexuais de $64,6 \%$ para $83,2 \%$ (BRASIL, 2018). Um assunto que vem ganhando destaque em pesquisas por ter se tornado uma das principais causas de morbimortalidade na atualidade no Brasil. 
Conforme a Resolução COFEN 564/2017, que aprova o Código de Ética dos profissionais de Enfermagem (CEPE), cabe ao profissional, cumprir a responsabilidade social e moral, independente da área que atua, de proteger, detectar, notificar qualquer caso de violência contra criança e adolescente. Possibilitando assim a prevenção a danos de uma pessoa que se encontra em situação de vulnerabilidade e proporcionando direito a uma vida saudável (ALGERI S e SOUZA LM, 2006).

Considerando a relevância das intervenções de enfermagem como uma forma de promover acolhimento, bem-estar e saúde em grupos vulneráveis como é o caso da criança e do adolescente vítima de abuso sexual, faz-se necessário conhecer as estratégias da assistência de enfermagem, bem como o nível de qualificação do profissional de enfermagem no enfrentamento do paciente vítima de abuso sexual.

Nesta perspectiva, este estudo tem como objetivo analisar de acordo com a literatura as repercussões do abuso e exploração sexual na criança e adolescente, a importância da qualificação profissional da enfermagem e da notificação frente as vítimas de violência sexual, para assim prestar um melhor atendimento e acolhimento à vítima.

\section{REVISÃO BIBLIOGRÁFICA}

\section{Repercussões do abuso e exploração sexual na criança e adolescente}

A violência e exploração sexual contra crianças e adolescentes tornou-se um problema de saúde pública, devido às consequências negativas do ato no desenvolvimento cognitivo, emocional, comportamental, físico e social dos indivíduos (HABIGZANG LF, et al., 2011).

A vivência em situações traumáticas pode levar a repercussões no seu desenvolvimento, desencadeando transtornos psicopatológicos, como transtorno de estresse pós-traumático (MILLER AB, et al., 2013), depressão e transtornos de ansiedade (HABIGZANG LF e CAMINHA RM, 2004), o que pode refletir na personalidade adulta.

Pesquisas apontam que o abuso sexual infantil é o facilitador para o surgimento de psicopatologias graves, prejudicando o desenvolvimento psicológico, afetivo e social da vítima. As sequelas da situação traumática podem se manifestar de várias maneiras, em qualquer idade da vida (ROMARO RA e CAPITÃO CG, 2007). Nos estudos de Karakurt G e Silver KE (2014) afirmam que as vítimas de violência sexual quando chegam à vida adulta se comportam com menos confiança nas relações com seus parceiros e sentem medo de abandono.

As consequências apresentadas pelas vítimas a curto e longo prazo depende dos fatores que compõem o abuso e exploração sexual, como: idade da criança, duração do abuso, sua frequência, envolvimento ou não de violência e ameaça, grau de relacionamento com o abusador, ausência de figuras protetoras. Tais causas podem atenuar ou intensificar o impacto da vivência (O'LEARY P, et al., 2010).

As sequelas mais comuns associadas a experiência do abuso sexual infantil podem estar relacionados a vergonha e ansiedade ( STEPHENSON KR, et al., 2012), depressão, baixa autoestima, insegurança e ideações suicidas, além de dificuldade de estabelecer relacionamentos duradouros, bem como incapacidade de evitar situações de revitimização (SANT'ANNA PA e BAIMA APS, 2008).

No âmbito de consequências orgânicas é possível notar lesões físicas gerais, lesões genitais, lesões anais, gestação, infecções sexualmente transmissíveis, disfunções sexuais, hematomas, contusões e fraturas (CUNHA EP, et al., 2008).

No contexto psicossocial, estudos mostram que as vítimas apresentam alterações, como: abusos de substâncias psicoativa, distúrbios do sono e alimentação, fuga do lar, pensamento de autodestruição, condutas sexualizadas ou delinquentes, prostituição, afastamento social, irritabilidade, dificuldade de confiança, baixo rendimento escolar, déficit de relacionamento afetivo, e sentimento de culpa, raiva e de diferença em relação aos iguais (COHEN JA, et al., 2001; CAMINHA RM, et al., 2003). As funções intelectuais e criativas também são afetadas e a criança para de brincar, perde o interesse pelos estudos, fechando-se em si mesma (HABIGZANG LF e CAMINHA RM, 2004). 
A vivência de abuso sexual pode comprometer o funcionamento sexual das vítimas, causando transtornos sexuais, tais como: disfunção sexual, aversão sexual e falta de prazer, falha de resposta genital incluindo impotência sexual, disfunção orogástrica, ejaculação precoce, vaginismo não orgânico, dispareunia não orgânica e a ninfomania.

Estudos apontam que as consequências do abuso podem manifestar em curto ou longo prazo, com indícios de alta atividade sexual, confusão sobre o gênero sexual, dificuldade no ajustamento sexual adulto, e confusão quanto aos valores sexuais (ROMARO RA e CAPITÃO CG, 2007). Podem ser desencadeados também transtornos de preferências sexuais, incluindo as parafilias, como: fetichismo, voyeurismo, sadomasoquismo, pedofilia, e outras, conforme descritas na Classificação de Transtornos Mentais e de Comportamento - CID - 10 (ORGANIZAÇÃO MUNDIAL DE SAÚDE, 1993).

A vivência a exploração sexual e suas sequelas têm sido debatido por diversos pesquisadores. Libório $R$ (2005) evidencia a dimensão processual do ato, propondo que a entrada na exploração é resultado de muitos fatores, que envolvem aspecto sociais, individuais e violações constante. Destaca-se na vítima o sentimento de: banalização, medo e vergonha, além da dificuldade em compreender o seu lugar de vítima na situação.

As sequelas da violência sexual podem se estender até a vida adulta, causando fragilidade e insegurança, menor tolerância a frustração, tornando-o indivíduo agressivo, e que o fenômeno pode ser recorrente nas gerações seguintes (SOMMER D, et al., 2017).

As repercussões do abuso e exploração sexual infanto-juvenil são extensas e diversas para a vítima, levando a consequências que refletem no futuro, além de gerar encargos ao sistema de saúde especificamente as consultas médicas e internações hospitalares, portanto é necessário compreender o quanto este ato pode desenvolver sérios problemas psicossociais e psicopatológico na vida do indivíduo, para que, assim se construam propostas de intervenções mais específicas no sentido de minimizar os danos dessa violência.

\section{A importância do preparo profissional para o acolhimento às vítimas de violência e exploração sexual}

Os profissionais de enfermagem compõem a equipe maior de uma unidade de saúde, responsáveis pela a triagem do atendimento, tendem a ter maior contato com o paciente e seus familiares, dessa forma assumindo uma função importante, pois através deste contato estabelecido podem identificar os sinais de violência sexual e, prevenir ou reduzir possíveis danos a vítima.

É através da anamnese, estabelecendo um diálogo com os responsáveis e a vítima que é possível coletar informações imprescindíveis para a caracterização do abuso, junto com o exame físico para determinar lesões e alterações decorrentes da violência, com a capacidade do profissional em ter um olhar holístico (WOISKI ROS e ROCHA DLB, 2010).

A atuação do enfermeiro frente ao abuso sexual requer conhecimento de toda semiologia e semiotécnica para perceber alterações na vítima e tempo de experiência para identificar qualquer tipo de suspeita de abuso contra o menor. É de competência do enfermeiro buscar o conhecimento específico, com a finalidade de adquirir o manejo adequado para prevenir e impedir o abuso, como promoção e proteção infanto-juvenil (MACHADO JC e VILELA ABA, 2018; PEDROSO LVB, 2014; VALERA IMA, et al., 2015).

Estudos apontam que os profissionais enfrentam diversas dificuldades, tais como: falta de capacitação e medo para atuar na prevenção e na notificação, despreparo para lidar com a vítima de violência sexual, falta de apoio e sigilo do conselho tutelar, desinteresse, desconhecimento, insegurança, medo do agressor, não saber para onde encaminhar a vítima, interferência emotiva entre o profissional e a vítima, sobrecarga de trabalho, deixando de protocolar as notificações compulsórias e de fazer denúncia anônima aos órgãos competentes.

Falta uma preparação destes profissionais para lidar com enfrentamento desta problemática, que é de suma importância o conhecimento e capacitação para avaliar o comportamento e o estado mental das vítimas, evitando assim uma falha no atendimento decorrente a falta de conhecimento e preparação para diagnosticar e dá uma assistência completa ao indivíduo (APOSTÓLICO MR, et al., 2013). 
Essa dificuldade de dar visibilidade à violência sexual infantil é muito complexa entre os profissionais de saúde, devido à falta de apoio ou até mesmo a inexistência dos órgãos competentes, estrutura emocional e o medo do provocador desse ato doloroso e cruel, o profissional sente-se intimidado a tomar providências cabíveis, trazendo como resultado, uma ineficaz assistência às vítimas e uma violência invisível (COCCO M, et al., 2010).

Portanto, o profissional de enfermagem necessita de um treinamento no serviço de atendimento à saúde, voltados a cursos de capacitação e direcionamento especializados para o cuidado adequado às vítimas, assim desenvolvendo habilidades imprescindíveis, como estar atento, saber reconhecer sinais e comportamentos suspeitos, enfrentando o problema de forma coerente, adotando uma postura profissional de responsabilidade social, com comprometimento de minimizar os danos, capacidade de identificar famílias de riscos e elaborar estratégias para acompanhá-las, promovendo meios para que 0 ato de abuso e exploração sexual não volte a acontecer (CUNHA EP, et al., 2008).

É de extrema importância a inclusão do tema abuso e exploração sexual na formação acadêmica do enfermeiro, uma vez que compete a universidade levantar a questão e levar aos profissionais o conhecimento da problemática, bem como preparo para lidar com situações diversas, sobretudo as mais recorrentes, como por exemplo, o abuso e exploração sexual infantil no nosso cotidiano (ALGERI S e SOUZA LM, 2006).

Desta forma formando um profissional capaz de compreender melhor todo o contexto, devolvendo para o ambiente um indivíduo qualificado, competente e socialmente comprometido no enfrentamento e combate da problemática em questão.

O preparo do enfermeiro foi mencionado como uma urgência para o efetivo enfrentamento da violência sexual e, por outro lado, a falta dessa capacitação é apontada como uma das causas da desordem entre a atuação do profissional e as necessidades das vítimas. As instituições de saúde e ensino superior são apontadas como responsáveis por promover essa qualificação, desde o estímulo a ações, como campanhas e eventos sobre o tema, até o apoio jurídico (SILVA MAI e FERRIANI MGC, 2007).

Estudos evidenciam que existe uma carência na formação acadêmica em contemplar os problemas do abuso e exploração sexual nas disciplinas que abrangem a criança, o adolescente e a família, um dos motivos dessa falta de abordagem se deve ao fato que estes temas é um problema/condição de saúde que não compreende os conceitos fisiopatológicos, não constando explicitamente no Código Internacional de Doenças- CID 10.

A inclusão curricular do assunto prepararia o enfermeiro, favorecendo a intervenção precoce dos cuidados e prevenção desse fenômeno, bem como capacitando o enfermeiro para a abordagem holística e humanizada, não focando seus cuidados exclusivamente para os danos físicos (ALGERI S e SOUZA LM, 2006).

O enfermeiro tem diversas funções, e uma delas dentro desta problemática é romper a violação dos direitos humanos, como educação na comunidade, prevenção, reabilitação e denunciar qualquer suspeita ou confirmação desta violência. Os profissionais que lidam com esta problemática devem ser acolhidos periodicamente para averiguar presença de sofrimento mental que estão enfrentando (ARAGÃO AS, et al., 2013).

O enfermeiro é peça fundamental na atuação contra o abuso e exploração infanto-juvenil, pois possui uma aproximação direta com a vítima, e precisa estar preparado para o enfrentamento de situações dolorosas, para assim ter a capacidade de intervir, prevenir, proteger, notificar e evitar qualquer recorrência contra a vítima. Ressalta-se, ainda, a necessidade de assegurar o encaminhamento das vítimas às redes de defesa e de assistência, com responsabilização social favorecendo uma melhor assistência a crianças e adolescentes vítimas de abuso e exploração sexual.

\section{A conduta do enfermeiro em relação a notificação diante do problema}

O conceito de notificação é entendido como ato de denunciar ou levar ao conhecimento das autoridades competentes a possibilidade de um eventual abuso. Segundo Parraga EE (2010) o termo denúncia é o ato de revelar ou alertar e, não de maneira acusatória pois a investigação é de competência das autoridades policiais 
e judiciárias. No que compete à enfermagem a notificação consiste em informar determinado setor ou órgão acerca dos maus tratos, abuso sexual ou qualquer outro tipo de violência que envolve menores de idade.

As notificações geralmente são feitas por parte da família para a polícia, que encaminham as vítimas para exame de corpo delito no IML (GUIMARÃES JATL e VILLELA WV, 2011). O ECA (Estatuto da Criança e do Adolescente) aponta como responsabilidade dos profissionais de saúde, notificar os casos que tiverem conhecimento e de acordo com Art.245, prevê penalidade para profissionais de saúde e educação que se recusem a denunciar os casos suspeitos ou confirmados (BRASIL, 2006).

Na Atenção Primária à Saúde (APS) as enfermeiras possuem uma vantagem para identificar os casos, pois adquirem vínculos com as crianças e familiares. Além de que o cuidado não deve ser pautado somente em tratamento clínico, visto que os efeitos negativos da violência causam repercussões na infância até sua vida adulta (CRUZ MA, et al., 2020).

A notificação constitui um indicador epidemiológico da violência, auxiliando o dimensionamento e redirecionamento de ações nos locais, bem como a formação de políticas públicas. Portanto, deve-se ter uma atenção especial ao encaminhamento das vítimas às redes de defesa e assistência. Para que tudo ocorra de forma completa, o profissional de saúde deve ser conscientizado da importância da notificação, e os cursos de graduação e formação profissional devem promover o aprimoramento na detecção e diagnóstico das situações de violência.

O fato que não denunciar pode ser compreendida como estratégia de proteção ao agressor, ocultando situações crônicas de violência, contribuindo para a subnotificação (BORGES JPA, 2014). É através da notificação que as políticas públicas e estratégias de enfrentamento da questão são criadas baseando-se no dimensionamento epidemiológico.

É frequente falhas que dificultam as notificações, como: dados incompletos, resultado de exames, dados do agressor, além da falta de conhecimento de quem acolhe, demonstrando a necessidade de política que acolha, compreendam, trate e haja de forma adequada, além de profissionais capacitados e protocolos para a situação. A produção científica sobre o tema é escassa, enfermeiros e outros profissionais de saúde possuem dificuldade na assistência quando se deparam com crianças e adolescente vitimadas por violência sexual, por falta de qualificação (RIBEIRO MA, et al., 2004).

Outro grande motivo pela falta de notificação, deve-se a falta de preparo inclusive na identificação. Uma pesquisa realizada com graduando de enfermagem, foi evidenciado que os cursos preparam profissionais para atuação diante sinais e sintomas físicos ou casos de lesões em corpo. Mas muitas crianças vitimizadas apresentam comportamentos, como agressão, irritabilidade, falta de envolvimento e socialização com outras crianças (MACHADO JC e VILELA ABA, 2018).

Num estudo realizado por Woiski ROS e Rocha DLB (2010), percebeu-se a comoção de profissionais de enfermagem que trabalham numa emergência, diante da constatação de uma criança vítima de violência sexual, algo incomum, onde geralmente existe a procura por motivos de traumas por acidentes ou problemas respiratórios e o agravante por se tratar de uma criança indefesa. Foi relatado por profissionais 0 distanciamento do enfermeiro e ausência de um protocolo diante tal situação.

Silva PA, et al. (2015) afirmam que há falta de divulgação em relação aos efeitos positivos da notificação pelos profissionais de enfermagem, fato esse que, somado à inexistência de protocolos específicos, ocasiona desânimo e negligência diante tal realidade. Portanto, as notificações com boas repercussões resultam em autoridade para os profissionais propiciando um obstáculo à violência.

Vale ressaltar que a notificação é uma das principais etapas do processo de enfrentamento da violência sexual infantil, visto que a partir dela derivam ações no âmbito das redes de atenção e proteção e estabelecimento de uma linha de acolhimento e cuidado às vítimas. O enfermeiro tem um importante papel nesse processo, capaz de perceber o abuso, notificar e orientar sua equipe para o enfrentamento e identificação, como registrar os dados corretos na base de dados da notificação, propiciando o melhor atendimento e proteção à vítima. Sendo que seu papel não se limita a notificação. 


\section{CONSIDERAÇÕES FINAIS}

O abuso e a exploração sexual infanto-juvenil, problema sofrido por crianças e adolescentes de todas as culturas, causam severas repercussões na vida da vítima como distúrbios comportamentais e emocionais na vítima que vão desde abuso de substâncias psicoativas ao pensamento autodestrutivo. A atuação do enfermeiro nesse processo perpassa desde a identificação dos casos, acolhimento das vítimas e sua família, cuidar da saúde biopsicossocial desses menores, além da notificação dos casos, requer deste profissional, conhecimentos, habilidades e ações estratégicas que promovam a redução de casos e proteção. Entretanto, nota-se um descompasso entre a atuação da enfermagem e as reais necessidades das vítimas, apontando como fatores responsáveis por esta realidade, a carência de capacitações oferecidas pelas instituições de saúde e a falta de abordagem desta temática no período acadêmico deste profissional. Sendo assim, é essencial e urgente a qualificação adequada do enfermeiro para assistir e acolher de forma humanística e holística essas vítimas, e logo notificar os casos corretamente.

\section{REFERÊNCIAS}

1. ALGERI S, SOUZA LM. Violência contra crianças e adolescentes: um desafio no cotidiano da equipe de enfermagem. Rev. Latino-Am. Enfermagem [periódico na Internet], 2006; 14(4): 625-631.

2. APOSTÓLICO MR, et al. As possibilidades de enfrentamento da violência infantil na consulta de enfermagem sistematizada. Revista da Escola de Enfermagem da Universidade de São Paulo, 2013; 320-327.

3. ARAGÃO AS, et al. Abordagem dos casos de violência à criança pela enfermagem na atenção básica. Revista Latino-Americana de Enfermagem, 2013; 21: 01-07.

4. ATALLAH NA e CASTRO AA. Revisão sistemática da literatura e metanálise: a melhor forma de evidência para tomada de decisão em saúde e a maneira mais rápida de atualização terapêutica [Internet],1997.

5. BRASIL. Conselho Federal de Enfermagem (COFEN). Resolução COFEN n.0564 de 06 dez 2017: Aprova o novo Código de Ética dos Profissionais de Enfermagem. 2017.

6. BRASIL. Ministério da Saúde. Secretária de Assistência à Saúde. Boletim Epidemiológico. Análise epidemiológica da violência sexual contra crianças e adolescentes no Brasil, 2011 a 2017, Brasília, DF, 2018; 49: 01-17.

7. BORGES JPA. Violência na Infância: perspectivas e desafios para a Enfermagem. Rev. Soc. Bras. Enferm. Ped. 2014; 14(2): 154-8.

8. CAMINHA RM, et al. Epidemiologia de abuso sexual infantil na clínica escola PIPAS/UNISINOS. Em Caderno de Extensão UNISINOS IV, 2003; 129-142.

9. COCCO M, et al. Abordagem dos profissionais de saúde em instituições hospitalares a crianças e adolescentes vítimas de violência. Rev. Eletr. Enf. [Periódico na Internet]. 2010; 12(3): 491-7.

10. COHEN JA, et al. Treatment practices for childhood posttraumatic stress disorder. Child Abuse \& Neglect, 2001; 25: 123-135.

11. CRUZ MA, et al. Repercussões do abuso sexual vivenciado na infância e adolescência: revisão integrativa. Cien Saude Colet, 2020.

12. CUNHA EP, et al. Enfrentamento à violência sexual infanto-juvenil: expansão do PAIR em Minas Gerais. Belo Horizonte: UFMG, 2008.

13. GUIMARÃES JATL e VILLELA WV. Características da violência física e sexual contra crianças e adolescentes atendidos no IML de Maceió, Alagoas, Brasil. Cad Saúde Pública, 2011; 27: 1647-53.

14. KARAKURT G e SILVER KE. Therapy for childhood sexual abuse survivors using attachment and family systems theory orientations. The American Journal of Family Therapy, 2014; 42(1): 79-91.

15. HABIGZANG LF, et al. A revelação de abuso sexual: Medidas adotadas pela rede de apoio. Psicologia: Teoria e Pesquisa, Brasília, 2011; 27: 467-473.

16. HABIGZANG LF e CAMINHA RM. Abuso sexual contra crianças e adolescentes: Conceituação e intervenção clínica. São Paulo: Casa do Psicólogo, 2004.

17. LEITE JT, et al. Enfrentamento da violência doméstica contra crianças e adolescentes na perspectiva de enfermeiros da atenção básica. Revista Gaúcha de Enfermagem, 2016; 37: 01-07.

18. LIBÓRIO R. Adolescentes em situação de prostituição: uma análise sobre a exploração sexual comercial na sociedade contemporânea. Psicologia: Reflexão e Crítica, 2005; 18(3), 413-420.

19. MACHADO JC e VILELA ABA. Conhecimento de estudantes de enfermagem na identificação de crianças em situação de violência doméstica. Revista de Enfermagem da Universidade Federal de Pernambuco, 2018; 12(1): 8390.

20. MILLER AB, et al. PTSD and marital satisfaction in military service members: Examining the simultaneous roles of childhood sexual abuse and combat exposure. Child Abuse \& Neglect, 2013; 37(11): 979-985.

21. MINISTÉRIO DA SAÚDE. Estatuto da Criança e do Adolescente.3rd. Ed. Brasília: Editora do Ministério da Saúde; 2006.

22. O'LEARY P, et al. The Effect of Severe Child Sexual Abuse and Disclosure on Mental Health during Adulthood. Journal of Child Sexual Abuse, 2010; 19(3): 275-289. 
23. ORGANIZAÇÃO MUNDIAL DE SAÚDE (OMS). Classificação de Transtornos Mentais e de Comportamento da CID10: descrições clínicas e diretrizes diagnósticas. Porto Alegre: Artes Médicas, 1993.

24. PARRAGA EE. O papel do enfermeiro no atendimento à criança e adolescente vítima da violência sexual. 2010. Monografia - Universidade Federal do Pampa, Uruguaiana, 2010.

25. PEDROSO VLB. Abuso sexual infantil: Conhecimento do enfermeiro sobre o seu papel no acolhimento das vítimas e na notificação de casos. 2013. 51 f. Dissertação (Mestrado em Psicologia) - Universidade Tuiuti do Paraná, Curitiba, 2014.

26. RIBEIRO MA, et al. Violência sexual contra crianças e adolescentes: características relativas vitimização nas relações familiares. Escola Nacional de Saúde Pública, Fundação Oswaldo Cruz, 2004.

27. ROMARO RA e CAPITÃO CG. As faces da violência: aproximações, pesquisas, reflexões. São Paulo: Vetor, 2007.

28. SANT'ANNA PA e BAIMA APS. Indicadores clínicos em psicoterapia com mulheres vítimas de abuso sexual. Psicologia: Ciência e Profissão, 2008: 28(4): 728-741.

29. SERPA MG. Primeiras experiências de exploração sexual: um estudo sobre o processo de aproximação de adolescentes a essa realidade. Revista PSICO, 2010; 41(1), 32-39.

30. SILVA PA, et al. Notificação da violência intrafamiliar contra crianças e adolescentes por profissionais de saúde no Brasil. Av en Enfermeria. 2015; 33(1): 142-50.

31. SILVA MAI e FERRIANI MGC. Violência doméstica: do visível ao invisível. Rev Latino-am Enfermagem,2007; 15(2).

32. SOMMER D, et al. Caracterização da Violência Contra Crianças e Adolescentes: Indicativos para a Prática do Enfermeiro. Revista de Enfermagem, 2017; 13: 14-28.

33. STEPHENSON KR, et al. Childhood sexual abuse moderates the association between sexual functioning and sexual distress in women. Child Abuse \& Neglect, 2012; 36(2): 180-189.

34. VALERA IMA, et al. Atuação da equipe de enfermagem diante da violência infantojuvenil. Revista Brasileira de Pesquisa em Saúde, 2015; 17(3): 103-111.

35. WOISKI ROS e ROCHA DLB. Cuidado de enfermagem à criança vítima de violência sexual atendida em unidade de emergência hospitalar. Esc Anna Nery Ver Enferm, 2010; 14: 143-150.

36. WORLD HEALTH ORGANIZATION (WHO). Guidelines for medico-legal care for victims of sexual violence. Geneva: 2003. 